\title{
Recent advances in genetic studies of stuttering
}

\author{
Changsoo Kang* \\ Department of Biology and Research Institute of Basic Sciences, Sungshin Women's University, Seoul, Korea
}

\begin{abstract}
Speech and language are uniquely human-specific traits, which contributed to humans becoming the predominant species on earth. Disruptions in the human speech and language function may result in diverse disorders. These include stuttering, aphasia, articulation disorder, spasmodic dysphonia, verbal dyspraxia, dyslexia and specific language impairment. Among these disorders, stuttering is the most common speech disorder characterized by disruptions in the normal flow of speech. Twin, adoption, and family studies have suggested that genetic factors are involved in susceptibility to stuttering. For several decades, multiple genetic studies including linkage analysis were performed to connect causative gene to stuttering, and several genetic studies have revealed the association of specific gene mutation with stuttering. One notable genetic discovery came from the genetic studies in the consanguineous Pakistani families. These studies suggested that mutations in the lysosomal enzyme-targeting pathway genes (GNPTAB, GNPTG and NAPGA) are associated with non-syndromic persistent stuttering. Although these studies have revealed some clues in understanding the genetic causes of stuttering, only a small fraction of patients are affected by these genes. In this study, we summarize recent advances and future challenges in an effort to understand genetic causes underlying stuttering.
\end{abstract}

Key words: Stuttering, Genetic linkage, Lysosomes, GNPTAB, GNPTG, NAGPA.

\section{Introduction}

Communication between human beings, mediated mostly by speech and language is a remarkable function that has had a significant influence on human life. Although speech and language are not easily distinguished, they are different from each other [1]. Speech is defined as the mechanical aspect of vocalization for communication, and includes articulation, voice and fluency. In contrast, language consists of non-mechanical syntactic rules such as grammar regulating the meaning of words, creation of new words, and application of words into meaningful combination to form a sentence (http://www.asha. org/public/speech/development/language_speech.htm). Both speech and language are regarded as unique features in humans that are not found in other species. Although it is well-known that many other animals can also communicate by generating vocalization, it is not entirely clear whether it has the syntactic rules. Human speech and language may be the most complex and well-organized form of vocalization compared with those in other animals $[2,3]$.

When individuals have speech deficits, they are regarded as being affected with speech disorders, and these include fluency disorders (e.g., stuttering), voice disorder (e.g., spasmodic dysphonia), articulation disorder, verbal dyspraxia and aphasia [4-6]. Among these speech disorders, stuttering is the most common speech or fluency disorder characterized by repetitions, prolongations, and interruptions in the smooth flow of the speech [4]. In the population, stuttering shows high

\footnotetext{
Received: 31 May 2015, Revised: 3 June 2015, Accepted: 4 June 2015, Published: 30 June 2015

*Corresponding author: Changsoo Kang, Ph.D.

Department of Biology and Research Institute of Basic Sciences, Sungshin Women's University, 55 Dobong-ro 76ga-gil, Gangbuk-gu, Seoul 142-732, Korea. Tel: +82-2-920-7638, Fax: +82-2-202-2047, E-mail: ckang@sungshin.ac.kr

Conflict of interest: I declare that we do not have any conflicts of interests.

(c) This is an open-access article distributed under the terms of the Creative Commons Attribution Non-Commercial License (http://creativecommons.org/licenses/by-nc/4.0/) which permits unrestricted non-commercial use, distribution, and reproduction in any medium, provided the original work is properly cited.

(c) Copyright 2015 by the Korean Society of Medical Genetics

www.e-kjgm.org
} 
spontaneous recovery rate, with higher occurrence rate in males than female subjects $[7,8]$. Similarly, singing and speaking in unison mitigate symptoms. These puzzling clinical features, and the fact that stuttering has its origin in the brain, hinder researchers from investigating the neural basis of stuttering. However, several genetic studies such as twin, adoption, and segregation studies have suggested that this disorder occurs by the inheritance of gene mutations [9-16]. Genetic evidence presented by several approaches, in particular, genome-wide linkage analysis have shed light on these causative genes.

Here, we describe outcomes from the past, current, and future challenges encountered in the genetic studies of speech and language disorders.

\section{Genetic Evidence of Stuttering}

Stuttering occur mostly in childhood. It affects up to 5\% of the population at the age of 3-4 years, with the male-to-female ratio at 2:1 at the preschool age, and changes to $4: 1$ at the age 9. This sex-ratio change is due to that majority of the stutters $(>75 \%)$ resolve spontaneously, especially in females $[7,8]$. Therefore, the estimated prevalence of stuttering is about $1 \%$ in the general population.

Consistent evidence for the involvement of genetic factors in stuttering has motivated studies aimed at identifying causative genetic mutations that could reveal underlying molecular and cellular deficits in this disorder. For example, twin studies revealed that concordance rate in monozygotic twins (63\%) was higher than that in the dizygotic twins (9\%) [9]. Half of the stutterers had a family history, and risk to first degree relative was 15\%. Several large families with multiple individuals were affected by stuttering [10-15].

In addition, there was debate that stuttering can be learned rather than inherited from their parents. Felsenfeld and Plomin [16] designed adoption study to disentangle nature and nurture in 156 adopted and non-adopted children, and estimated their risk for stuttering based on parental history of stuttering. It was revealed that children with biological parents who stuttered showed 2.8 times higher risk for developing persistent stuttering than those with no parental history of stuttering. Thereby, implying that stuttering is not a learned behavior.

Based on a previous twin, adoption study, and the fact that stuttering runs in the family, it was strongly suggested that genetics may play an important role in the degree of susceptibility to stuttering. This motivated geneticists to perform genome-wide linkage analysis with the purpose to identify causative genes. Identifying the mode of inheritance and potential number of causative genes (monogenic or polygenic) were prerequisites for successful study of stuttering. Cox et al. [17] performed segregation analysis in 386 stutterers and their first-degree relatives to investigate precise model of transmission, and reported that genetic causes of stuttering cannot be attributed to single major locus, nor follow typical Mendelian mode of inheritance. Although there is substantial evidence that genetic factors are involved in susceptibility to stuttering, the suggested genetic model was found to be inconclusive.

\section{Genome-wide Linkage Analysis}

\section{Genetic studies in outbred stuttering families}

Despite the unclear mode of inheritance, multiple genomewide linkage studies were performed in an effort to identify chromosomal loci or genes in families affected by stuttering. The first linkage scan for stuttering was carried out by the National Institute on Deafness and Other Communication Disorders (NIDCD) at the National Institutes of Health (NIH). Shugart et al. [18] recruited 68 stuttering families with European Ancestry, and diagnosed affected individuals by recording both their conversation and reading skills [18]. Whole-genome scan using 392 microsatellite markers analyzed by GENEHUNTER and ALLEGRO programs found suggestive linkage (non-parametric linkage [NPL] score: 1.51) at D18S976 on chromosome 18p. Subsequent analysis with the single largest family alone revealed an NPL score of 5.35 , which suggested that chromosome $18 p$ is a predisposing locus for stuttering. Although this was the first whole-genome linkage analysis, further analysis to identify the causative gene in this locus remains to be performed.

Other genetic linkage studies by NIDCD/NIH applied to a single large Cameroonian family comprising 71 individuals, and to a group of 43 Brazilian families, reported genome-wide significant linkage of stuttering to the markers on chromosomes $2 p, 15 q$ (Cameroonian family: logarithm of odds [LOD] score $=4.69-6.57$ ) and 10q21 (Brazilian family, LOD score=4.28).

In addition, another research team at The Illinois International Genetics of Stuttering Project, University of Chicago also performed several whole-genome linkage analyses to identify genetic causes of stuttering. They recruited 100 families including 252 individuals of European ancestry, and found moderate linkages of stuttering to the microsatellite markers on multiple chromosomes $2,7,9,15$, and 21. Interestingly, sex-specific significant linkage scores were also reported in 
this study. In the analysis with male subjects only, linkage was found on markers at chromosome 7 (LOD score $=2.99$ ) while female-only analysis revealed linkage at chromosome 21 (LOD score $=4.5$ ). This sex-specific linkage results met the criteria for genome-wide significance [18]. Another interesting linkage study performed in a Hutterite population characterized by presence of relationship in a single genealogy with 232 individuals sharing the same ancestor, reported modest evidence of linkage on chromosomes 2, 3, 5, 13, and 15 [19].

Although tremendous effort was put into these linkage and association studies, they were limited in that they only reported chromosomal loci, and were unable to find any particular candidate genes for stuttering. Targeted deep-sequencing using next-generation sequencing on these linkage intervals need to be performed to investigate causative mutations in the near future.

\section{Genetic studies in inbred stuttering families}

All genome-wide linkage studies described above were in families from outbred populations until NIDCD/NIH group led by Drayna et al. [13] studied Pakistani inbred families affected by stuttering. In genetic studies of rare Mendelian disorders, consanguineous families were regarded as a promising starting point for success. Using a Pakistani family in genetic studies is advantageous because $~ 70 \%$ of all marriages are between either 1st or 2nd cousins. Consanguineous marriages result in a population structure with greatly increased incidence of recessive genetic disorders. This might be true for complex traits such as stuttering.

The first genome-wide linkage study of stuttering in Pakistani inbred family was performed by Riaz et al. [13] at NIDCD/NIH. In this study, forty-four families with multiple individuals affected by stuttering were ascertained from the city of Lahore and nearby areas in Pakistan. The status of stutterers was diagnosed using the Stuttering Severity Instrument, 3rd edition (SSI3). Genome-wide linkage scan using microsatellite marker panel found significant linkage of stuttering to the markers at chromosome 12q23.3 (LOD score $=4.61$ ), which suggested that this significant linkage was mostly attributed to the largest family named PKST72 [13]. The linkage interval at chromosome 12q23.3 expands 10 megabases with PAH marker at the center, and eighty seven genes reside in this locus.

In an effort to follow-up on the linkage study of Pakistani inbred family, Kang et al. [14] performed comparative genomic hybridization to exclude possible presence of large insertional or deleterious (in/del) mutation in the affected individuals in this family. They concluded that there was no evidence of affected members carrying any large in/del mutation co-segregating with stuttering. Sanger sequencing of genes in this linkage interval revealed a missense mutation replacing glutamate with lysine residue at position 1,200 (p.Glu1,200Lys) in GlcNAc1 -phosphotransferase $\alpha / \beta$, encoded by GNPTAB. Further sequencing of GNPTAB gene in the 123 unrelated Pakistani stutters revealed that eight individuals (35\%) were carrying this mutation, but this was not observed in Caucasian and African populations [14]. This population-specific observation of p.Gul1200Lys mutation in GNPTAB raised the possibility that this might be a founder mutation only in the Pakistani population. Haplotype analysis surrounding the GNPTAB region showed that this was a founder mutation with an estimated age of about 572 generations or 14,300 years old [20].

Two other proteins, GIcNAc-1-phosphotransferase $\gamma$ (encoded by GNPTG) and GIcNAc-1-phosphodiester $\alpha-\mathrm{N}$ acetylglucosaminidase (encoded by NAGPA) were regarded as potential candidate genes for stuttering because their functions are closely related with GNPTAB. Products of GNPTG and NAGPA, together with GNPTAB are involved in protein trafficking from Golgi complex to endoplasmic reticulum (ER) (Fig. 1). Further sequencing of GNPTG and NAGPA found six mutations (three in each gene) in the unrelated North American stutterers [14]. Among them, four mutations were missense and the other two were insertion (GNPTG: p.Leu5_Arg7Dup) and deletion (NAGPA: p.Phe513SerfsX113) mutations. All these mutations result in small changes in the amino acid sequence.

\section{Lysosomal Enzyme-targeting Pathway and Stuttering}

GlcNAc-1-phosphotransferase (EC2.7.8.17) is a hexameric complex consisting of three different subunits ( 2 alpha, 2 beta and 2 gamma subunits). Alpha and beta subunits are encoded by GNPTAB and gamma is encoded by another gene GNPTG [21]. This enzyme plays a role in the initial step in the tagging of mannose-6-phosphate signal required for transport of newly synthesized lysosomal enzymes from Golgi complex to ER (Fig. 1). Loss of enzyme function is well known for causing rare lysosomal storage disorders, mucolipidoses types II (OMIM \# : 252500) and III (OMIM \#: 252605) [22-25]. Mucolipidose is a life-threatening fatal disorder, characterized by the presence of psychomotor retardation, clear corneas, restricted joint movement and congenital dislocation of body including hip and chest. In addition, one phenotype observed in mucolipidoses 


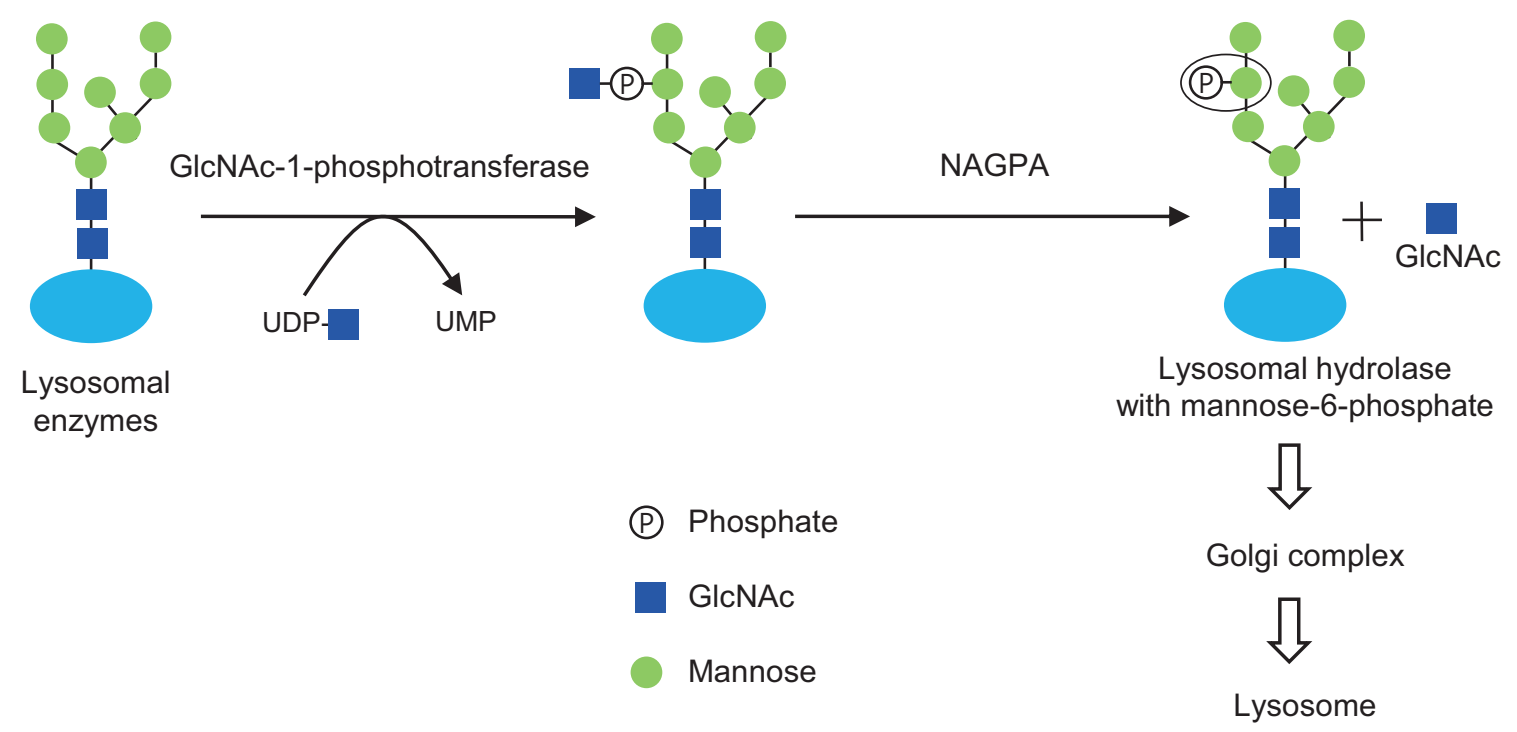

Fig. 1. Lysosomal enzyme targeting pathway. Adding mannose-6-phosphate tag is mediated by two step procedures mediated by two enzymes, GIcNAc-1-phosphotransferase (encoded by GNPTAB and GNPTG) and N-acetylglucosamine-1-phosphodiester alpha- $\mathrm{N}$-acetylglucosaminidase (encoded by NAGPA). In the first step, GIcNAc-1-phosphotransferase catalyze adding GlcNAc-1-phosphate to the terminal mannose residue of the oligosaccharides on lysosomal enzymes destinied to lysosome. In the second step, $\mathrm{N}$-acetylglucosamine-1-phosphodiester alpha-Nacetylglucosaminidase cleave off GIcNAc residue. The resulting lysosomal enzymes with mannose-6-phosphate tag are transported to the lysosome through the Golgi complex. UDP, uridine diphosphate; UMP, uridine monophosphate.

patients is that they often have deficit in speech function including stuttering, but this has been regarded as subsidiary phenotype due to developmental delay $[23,26]$. Therefore, it did not seem plausible to expect the association of mutations in GNPTAB or GNPTG with persistent stuttering. However, Kang et al. [14] proposed that disruptions in the lysosomal enzymetargeting pathway were associated with stuttering.

Another question to be addressed includes why individuals with mutations in GNPTAB or GNPTG only show stuttering without any other symptoms commonly found in mucolipidoses patients. This was explained by comparing the mutation spectrum of GNPTAB and GNPTG from stutters with those from mucolipidoses patients. Missense mutations were predominantly found in non-syndromic persistent stutters, in contrast to the fatal frameshift or stop codon mutations that resulted in complete lost-of-function in the mucolipidoses patients [14,2325]. This finding suggests that different types of mutation in the same gene results in different diseases due to the diverse level of enzyme activities.

GIcNAc-1-phosphodiester- $N$-acetylglucosaminidase (uncovering enzyme; EC 3.1.4.45), encoded by NAGPA is also reported to be associated with stuttering [14]. This enzyme is well known for catalyzing the second step in the synthesis of mannose-6-phosphate tag on lysosomal hydrolases destined to ER from trans-Golgi [27]. There was no report of human diseases associated with NAGPA until Kang et al. [14] found mutations in this gene from unrelated stuttering individuals. Mutant NAGPA from stuttering individuals showed lower cellular enzymatic activity (about 50\% of wild-type) [28].

The mutant NAGPA was not properly folded and localized in the ER rather than Golgi where normal NAGPA are mostly localized. Furthermore, this misfolded NAGPA was sensitive to degradation by proteasomal systems in the ER [28].

While data on GlcNAc-1-phosphotransferase remains limited due to the absence of functional assays, studies on the NAGPA enzyme showed that mutation reduced its enzymatic activity by about half. This resulted in disruptions in intracellular trafficking that led to a reduced half-life of the enzyme. However, it is still unclear how disruption in the lysosomal enzyme-targeting pathway affects the speech function of the brain, thereby causing stuttering.

\section{Future Challenges and Conclusion}

Recent genetic studies of stuttering within families and sporadic cases revealed multiple chromosomal loci linked with this disorder. Linkage analysis, particularly in the Pakistani inbred families revealed that mild disruption in the lysosomal enzyme-targeting pathway mediated by GNPTAB, GNPTG, and NAGPA are associated with persistent stuttering, and are the 
causative genes for stuttering. Disruption in this pathway may be attributed to 2-4\% of the affected individuals, and conclusive genetic causes of stuttering remains unclear. To accelerate the finding of further causative genes, next-generation sequencing technologies, such as whole-exome or targeted sequencing need to be applied.

Another challenge is the development of suitable animal model system for stuttering studies. In the pipeline of genetic discovery, phenotype should be observed in the animal model when mutations found in human patients are introduced into the genome of the animal. However, human speech and language are human-specific traits, thus, stuttering phenotype is not observable in the animal model. Despite this uncertainty, the mouse model is known for communicating ultrasonic vocalization. Analysis and comparison of vocalization patterns of wild-type to knock-in/out mouse might be a potent approach to study the association of lysosomal enzyme-targeting pathway and stuttering.

\section{Acknowledgements}

This study was supported by the intramural grant from Sungshin Women's University (2013-1-11-062/1).

\section{References}

1. Kang C, Drayna D. Genetics of speech and language disorders. Annu Rev Genomics Hum Genet 2011;12:145-64.

2. Holy TE, Guo Z. Ultrasonic songs of male mice. PLoS Biol 2005;3:e386.

3. White SA, Fisher SE, Geschwind DH, Scharff C, Holy TE. Singing mice, songbirds, and more: models for FOXP2 function and dysfunction in human speech and language. J Neurosci 2006;26:10376-9.

4. Bloodstein O. A handbook on stuttering. 6th ed. Clifton Park, NY: Thomson Delmar Learning, 2008.

5. Cole M, Wright D, Banker BO. Familial aphasia: the Pick-Alzheimer spectrum. Trans Am Neurol Assoc 1979;104:175-9.

6. Lera G, Bhatia K, Marsden CD. Dystonia as the major manifestation of Leigh's syndrome. Mov Disord 1994;9:642-9.

7. Drayna D, Kilshaw J, Kelly J. The sex ratio in familial persistent stuttering. Am J Hum Genet 1999;65:1473-5.

8. Dworzynski K, Remington A, Rijsdijk F, Howell P, Plomin R. Genetic etiology in cases of recovered and persistent stuttering in an unselected, longitudinal sample of young twins. Am J Speech Lang Pathol 2007;16:169-78.

9. Howie PM. Concordance for stuttering in monozygotic and dizygotic twin pairs. J Speech Hear Res 1981;24:317-21.
10. Raza MH, Amjad R, Riazuddin S, Drayna D. Studies in a consanguineous family reveal a novel locus for stuttering on chromosome 16q. Hum Genet 2012;131:311-3.

11. Raza MH, Gertz EM, Mundorff J, Lukong J, Kuster J, Schäffer AA, et al. Linkage analysis of a large African family segregating stuttering suggests polygenic inheritance. Hum Genet 2013;132:385-96.

12. Raza $M H$, Riazuddin S, Drayna D. Identification of an autosomal recessive stuttering locus on chromosome 3q13.2-3q13.33. Hum Genet 2010;128:461-3.

13. Riaz N, Steinberg S, Ahmad J, Pluzhnikov A, Riazuddin S, Cox NJ, et al. Genomewide significant linkage to stuttering on chromosome 12 Am J Hum Genet 2005;76:647-51.

14. Kang $C$, Riazuddin S, Mundorff J, Krasnewich D, Friedman P, Mullikin $\mathrm{JC}$, et al. Mutations in the lysosomal enzyme-targeting pathway and persistent stuttering. N Engl J Med 2010;362:677-85.

15. Suresh $R$, Ambrose $N$, Roe $C_{1}$ Pluzhnikov A, Wittke-Thompson JK, Ng $M C$, et al. New complexities in the genetics of stuttering: significant sex-specific linkage signals. Am J Hum Genet 2006;78:554-63.

16. Felsenfeld S, Plomin R. Epidemiological and offspring analyses of developmental speech disorders using data from the Colorado Adoption Project. J Speech Lang Hear Res 1997;40:778-91.

17. Cox NJ, Kramer PL, Kidd KK. Segregation analyses of stuttering. Genet Epidemiol 1984;1:245-53.

18. Shugart YY, Mundorff J, Kilshaw J, Doheny $K$, Doan B, Wanyee J, et al. Results of a genome-wide linkage scan for stuttering. Am J Med Genet A 2004;124A:133-5.

19. Wittke-Thompson JK, Ambrose $N_{1}$ Yairi E, Roe $C_{1}$ Cook EH, Ober $C_{1}$ et al. Genetic studies of stuttering in a founder population. J Fluency Disord 2007;32:33-50.

20. Fedyna A, Drayna $D$, Kang C. Characterization of a mutation commonly associated with persistent stuttering: evidence for a founder mutation. J Hum Genet 2011;56:80-2.

21. Bao M, Booth $J \mathrm{~L}$, Elmendorf BJ, Canfield WM. Bovine UDP-Nacetylglucosamine:lysosomal-enzyme N-acetylglucosamine-1phosphotransferase. I. Purification and subunit structure. J Biol Chem 1996:271:31437-45

22. Kornfeld S. Trafficking of lysosomal enzymes in normal and disease states. J Clin Invest 1986;77:1-6.

23. Otomo T, Muramatsu T, Yorifuji T, Okuyama T, Nakabayashi H, Fukao T, et al. Mucolipidosis II and III alpha/beta: mutation analysis of 40 Japanese patients showed genotype-phenotype correlation. J Hum Genet 2009;54:145-51.

24. Paik KH, Song SM, Ki CS, Yu HW, Kim JS, Min KH, et al. Identification of mutations in the GNPTA (MGC4170) gene coding for GICNAcphosphotransferase alpha/beta subunits in Korean patients with mucolipidosis type II or type IIIA. Hum Mutat 2005;2:308-14. 
25. Tiede $S$, Storch $S$, Lübke $T$, Henrissat $B$, Bargal $R$, Raas-Rothschild $A$, et al. Mucolipidosis II is caused by mutations in GNPTA encoding the alpha/beta GlcNAc-1-phosphotransferase. Nat Med 2005;11:110912.

26. Smuts I, Potgieter D, van der Westhuizen FH. Combined tarsal and carpal tunnel syndrome in mucolipidosis type III. A case study and review. Ann N Y Acad Sci 2009;1151:77-84.
27. Do H, Lee WS, Ghosh $P_{1}$ Hollowell T, Canfield W, Kornfeld S. Human mannose 6-phosphate-uncovering enzyme is synthesized as a proenzyme that is activated by the endoprotease furin. J Biol Chem 2002;277:29737-44.

28. Lee WS, Kang C, Drayna D, Kornfeld S. Analysis of mannose 6-phosphate uncovering enzyme mutations associated with persistent stuttering. J Biol Chem 2011;286:39786-93. 